\title{
Timing the Eccentric Binary Millisecond Pulsar in NGC 1851
}

\author{
Paulo C. C. Freire ${ }^{1}$, Scott M. Ransom² ${ }^{2}$ Yashwant Gupta ${ }^{3}$
}

\begin{abstract}
We have used the Green Bank Telescope to observe the millisecond pulsar PSR J0514-4002A on 43 occasions spread over 2 years. This 5-ms pulsar is located in the globular cluster NGC 1851; it belongs to a binary system and has a highly eccentric $(e=0.888)$ orbit. We have obtained a phase-coherent timing solution for this object, including very precise position, spin and orbital parameters. The pulsar is located 4".6 (about 1.3 core radii) from the center of the cluster, and is likely to lie on its more distant half. The non-detection of eclipses at superior conjunction can be used, given the peculiar geometry of this system, to rule out the possibility of an extended companion. We have measured the rate of advance of periastron for this binary system to be $\dot{\omega}=0.01289(4)^{\circ} \mathrm{yr}^{-1}$, which if due completely to general relativity, implies a total system mass of $2.453(14) \mathrm{M}_{\odot}$. Given the known mass function, the pulsar mass has to be $<1.5 \mathrm{M}_{\odot}$ and the mass of the companion has to be $>0.96 \mathrm{M}_{\odot}$, implying that it is a heavy white dwarf. The $350-\mathrm{MHz}$ flux density of this pulsar varies between 0.2 and $1.4 \mathrm{mJy}$; the origin of these variations is not known.
\end{abstract}

Subject headings: binaries: general — pulsars: general — pulsars: individual (PSR J0514-4002A) — globular clusters : general — globular clusters: individual (NGC 1851)

\section{Introduction}

PSR J0514-4002A was discovered in a 327-MHz search for steep-spectrum pulsars in globular clusters (GCs) carried out with the Giant Metrewave Radio Telescope (GMRT) at

\footnotetext{
${ }^{1}$ National Astronomy and Ionosphere Center, Arecibo Observatory, HC3 Box 53995, PR 00612, USA; pfreire@naic.edu

${ }^{2}$ National Radio Astronomy Observatory, 520 Edgemont Road, Charlottesville, VA 22903, USA; sransom@nrao.edu

${ }^{3}$ National Centre for Radio Astrophysics, P.O. Bag 3, Ganeshkhind, Pune 411007, India; ygupta@ncra.tifr.res.in
} 
Khodad, near Pune, India (Freire et al. 2004, henceforth Paper I). The pulsar is located in the globular cluster NGC 1851 and we will hereafter designate it to be NGC 1851A. It has a spin frequency of $200 \mathrm{~Hz}$ and is a member of a binary system, with a massive $\sim 1 \mathrm{M}_{\odot}$ companion and a very eccentric orbit $(e=0.888)$.

In the Galaxy, all MSPs are either found isolated or in low-eccentricity binary systems with low-mass white dwarfs (WDs). There are pulsars with eccentric orbits and massive companions, but their spin periods are tens, not hundreds, of Hz. For these systems, there is not much time for accretion before the massive companion becomes a neutron star (see Lorimer, 2005 and references therein).

NGC 1851A has hybrid characteristics. Its fast rotation indicates that the pulsar itself probably formed in the same manner as a normal MSP, ending up with a light WD companion in a nearly circular orbit. However, a subsequent stellar interaction could have disrupted the original binary system, replacing the lowest mass component (the original WD) with the present companion star in a highly eccentric orbit. Such exchange interactions are only likely to happen in environments with very high stellar densities, like the central regions of globular clusters. The same process could also form even more exotic systems, such as MSP/MSP or MSP/Black Hole binaries.

An alternative scenario for the formation of such binaries is the nearly head-on collision

of a neutron star (possibly even an MSP) with a giant star (e.g. Rasio \& Shapiro 1991; Lombardi et al. 2006); this can form an e $=0.9$ binary system consisting of the MSP and the stripped core of the giant star. We discuss this possibility at length in section $\$ 5$, since it might provide an explanation for the unusual flux density variations observed for this system.

In Paper I, we had limited information to constrain the nature of the companion star, no mass estimates for either the pulsar or the companion, and no precise pulsar timing solution. In order to correct these deficiencies, we observed the pulsar on 43 occasions over the last two years with the Green Bank Telescope, and we present the results below.

\section{Observations and data reduction}

\subsection{Data taking}

When observing NGC 1851A, we used mostly the prime-focus receiver PF1 with the 350$\mathrm{MHz}$ feed. On several occasions, however, we used the PF1 receiver with the 425- and 820$\mathrm{MHz}$ feeds, and twice used S-band receiver. The back-end instrument was the Pulsar Spigot 
(Kaplan et al. 2005). For the observations at frequencies below $1 \mathrm{GHz}$, where the pulsar is clearly detectable, we observed a total bandwidth of $50 \mathrm{MHz}$, divided in 1024 channels, with a sampling time of $81.92 \mu \mathrm{s}$. The S-band observations had a bandwidth of $600 \mathrm{MHz}, 768$ channels, and $81.92 \mu \mathrm{s}$ sampling. The data were dedispersed and folded using the PRESTO software packag@ 1 , resulting in 64 -bin pulse profiles $(\sim 77 \mu$ s per bin) approximately every $500 \mathrm{~s}$.

With the pulsar's dispersion measure (DM) of $52.14 \mathrm{pc} \mathrm{cm}^{-3}$, the $350 \mathrm{MHz}$ observations had $492 \mu$ s of dispersive smearing in each channel, giving an effective system time resolution of $499 \mu \mathrm{s}$. At this frequency, dispersive smearing is the dominant contribution to the width of the Gaussian-like pulse profile, which is $\sim 590 \mu$ s at half-power. At $425 \mathrm{MHz}$ the dispersive smearing is $275 \mu \mathrm{s}$ and the total time resolution is $287 \mu \mathrm{s}$, compared to the observed pulse width of $\sim 370 \mu \mathrm{s}$. Therefore dispersive smearing is still the dominant contribution to the pulse width at $425 \mathrm{MHz}$. These measurements suggest that the use of coherent dedispersion would significantly improve the timing precision of this pulsar.

\section{2. $\quad$ Timing}

We cross-correlated the average pulse profiles with a Gaussian template in the Fourier domain (Taylor 1992) to obtain topocentric times of arrival (TOAs). These were then analyzed with TEMPd2, using the DE 405 Solar System ephemeris (Standish 1998) to model the motion of the Green Bank Telescope relative to the Solar System Barycenter.

After an initial fit, we obtained a $\chi^{2}$ per degree of freedom of 1.5. Because there are no unmodeled trends in the residuals (their distribution is Gaussian), we believe that the routine used to derive the TOAs is slightly under-estimating the TOA uncertainties. After increasing the TOA uncertainties by $22 \%$ (a value similar to what we have seen for isolated MSPs timed with the same software) we obtained a $\chi^{2}$ per degree of freedom of 1.0 . The resulting timing parameters are presented in Table 2, the uncertainties are the 1- $\sigma$ TEMPO estimates. The significance of these timing parameters is discussed below.

The orbital parameters were determined using the Damour \& Deruelle orbital model (Damour \& Deruelle 1985; Damour \& Deruelle 1986). We measured the longitude of periastron $(\omega)$ and the time of periastron passage $\left(T_{0}\right)$ at a time (August 2005) where we have a sequence of daily observations that cover one full orbit. That $T_{0}$ was chosen as the reference

${ }_{1}^{1}$ http://www.cv.nrao.edu/\$\sim\$sransom/presto/

2 http://pulsar.princeton.edu/tempo/ 
epoch. The relativistic parameter $\gamma$, if included in the model, is very strongly correlated with the projected semi-major axis $x$ and $\omega$. When $\gamma$ eventually becomes needed by the timing model, the measured $x$ and $\omega$ will not change by more than $0.007 \mathrm{~s}$ and $0.09^{\circ}$, respectively, from their present values.

\section{Position, period and period derivative}

In Paper I, the position of the source identified as the pulsar in an interferometric map of the cluster is given as $\alpha=05^{\mathrm{h}} 14^{\mathrm{m}} 06.74 \pm 0.06^{\mathrm{s}}, \delta=-40^{\circ} 02^{\prime} 50.0 \pm 1.3^{\prime \prime}(\mathrm{J} 2000)$. The $\alpha$ and $\delta$ given in Table 2 are 1- $\sigma$ consistent with these values, but much more precise. The pulsar is about 4".6 (about 1.3 core radii) East of the center of the cluster (see Table 1). Such close proximity to the center is typical for GC pulsars 3 due to the effects of mass segregation. The uncertainty in the derived magnitude of the projected offset from the center of the cluster is dominated by uncertainties in the position of the cluster center and the cluster's core radius.

The observed period derivative, $\dot{P}_{\text {obs }}$, is the sum of several different terms (Phinney 1992):

$$
\left(\frac{\dot{P}}{P}\right)_{\mathrm{obs}}=\left(\frac{\dot{P}}{P}\right)_{\mathrm{int}}+\frac{1}{c}\left(a_{G}+\mu^{2} D+a_{c}\right),
$$

where $\dot{P}_{\text {int }}$ is the intrinsic period derivative of the pulsar, $a_{G}$ is the difference in Galactic accelerations between the Solar System Barycenter and NGC 1851 projected along the lineof-sight, $\mu$ and $D$ are the proper motion and distance to the pulsar (assumed to be the same as the cluster values in Table 1) and $a_{c}$ is the acceleration of the binary caused by the cluster, also projected along the line-of-sight. For $a_{G}$, we obtain $6.1 \times 10^{-12} \mathrm{~ms}^{-2}$ (Kuijken \& Gilmore 1989). For $\mu^{2} D$, we obtain $6.5 \times 10^{-11} \mathrm{~m} \mathrm{~s}^{-2}$, using the value for $\mu$ obtained by Dinescu et al. (1999). With these values and $\dot{P}_{\text {obs }}$ we obtain:

$$
\dot{P}_{c} \equiv \dot{P}_{\text {int }}+P \frac{a_{c}}{c}=(+0.0 \pm 1.4) \times 10^{-22}
$$

Pulsars with negative $\dot{P}_{c}$ can put lower limits on the absolute value of $a_{c}$, and for that reason have been used to study the gravitational potential of their parent GCs (e.g., Possenti et al. 2002, Freire et al. 2003). In this case, that can't be done, but we can say that it is very likely that the pulsar is located on the more distant half of the cluster. If it were on the

${ }^{3}$ See, e.g., http://www2.naic.edu/\$\sim\$pfreire/GCpsr.html 
near side, then $a_{c}$ in eqn. 2 would be positive, and $\dot{P}_{\text {int }}<2.8 \times 10^{-22}(2-\sigma$ upper limit). This would imply an anomalous characteristic age of more than 280 Gyr. In the likely event that the pulsar has a more normal characteristic age, then $a_{c}$ has to be negative, placing the pulsar on the far side of the cluster.

In Table 1, $v_{z}(0)$ is the spread of stellar line-of-sight velocities at the core. Using this, the cluster core radius and an analytical gravitational model for the central regions of GCs (Freire et al. 2005), we can calculate the maximum negative acceleration that the cluster can induce on the pulsar at its sky position. From this we can derive an upper limit on the intrinsic period derivative and surface magnetic flux density, and a lower limit on the characteristic age (see Table 2), as assumed in the formation scenarios in Paper I.

\section{On the nature of the companion}

\subsection{Is the companion extended?}

In Paper I, we reached the conclusion that, in the event of an extended companion, the circularization timescale for this system is larger than the age of the cluster. An extended companion could not therefore be disproved because of the system's eccentricity.

In Table 3, we present results of calculations of the companion masses, absolute component separation at superior conjunction $\left(a_{\text {sup }}\right)$ and its projection in the plane of the sky $\left(S=a_{\text {sup }} \cos i\right.$ ) for a set of orbital inclinations. The companion masses are calculated for a pulsar mass of $1.2 \mathrm{M}_{\odot}$; the mass of the lightest known NS, the companion of PSR J1756-2251 (Faulkner et al. 2005, Ferdman et al. 2006). For these companion masses, we list the radii of zero-age main-sequence stars, calculated using $\log _{10}\left(R / R_{\odot}\right)=0.917 \times \log _{10}\left(M / M_{\odot}\right)-0.020$, which apply for stars of mass similar to that of the Sun (Allen \& Cox 2000). The use of the smallest possible pulsar mass and corresponding zero age main sequence radii leads to the lowest possible companion masses and (if extended) sizes, the most conservative assumption in the discussion that follows.

For $i<60^{\circ}$ (limits are approximate), the companion masses are above $1 \mathrm{M}_{\odot}$. In a star cluster as old as NGC 1851 (about 9 Gyr, see Tab. 1), such stars have either finished their life cycles and left compact remnants behind, or are now in their giant phases. Giant stars have radii that are tens to hundreds of times that of similarly massive stars on the main sequence, which are of the order of one solar radius. Such giant stars would not even fit within the distance between the components of NGC $1851 \mathrm{~A}$ at superior conjunction or

periastron. In the first scan taken in 2006 July 7, we can detect the pulsar clearly through periastron, implying that the possibility of such a giant companion is excluded. 
For $i>80^{\circ}$, the radius of a main-sequence companion star is larger than $S$, implying that an eclipse should occur. The longitude of periastron for this system is $82.27^{\circ}$, and therefore superior conjunction occurs at a true anomaly of $7.73^{\circ}, 15 \mathrm{~min}$ and $54.1 \mathrm{~s}$ after periastron. For the second scan taken on 2006 July 7, the two TOAs occur 11.3 and 22.5 min of barycentric time after the periastron 4 . The pulsar is detectable throughout the whole scan. This excludes the possibility of the companion being a main sequence star with a mass smaller than $0.87 \mathrm{M}_{\odot}$.

For $60^{\circ}<i<80^{\circ}$, we could in principle avoid eclipses and still postulate a mainsequence companion. For any higher pulsar mass, or extended stars larger than zero age main-sequence, this inclination interval becomes smaller. However, even for the lowest inclination listed in Table 3, the pulsar signal would be passing only $1.2 \mathrm{R}_{\odot}$ above the surface of the companion, right through its corona. We also note that the pulsar wind should lead to a denser and more extended corona in this star, so we should be able to detect some increase in the electron column density at this orbital phase. Dividing the second observation of 2006 July 7 in 4 sub-bands and calculating TOAs for the whole observation, we obtain a DM of $52.148(5) \mathrm{pc} \mathrm{cm}^{-3}$, not measurably different than the DM at other orbital phases and smaller than the increases in the plasma column density seen at superior conjunction for pulsars with extended companions (see Freire, 2005 and references therein).

Furthermore, if the companion was extended, there would be tidal effects on the orbit of the pulsar (e.g., PSR J0045-7319, Lai 1996 and PSR B1259-63, Wang et al. 2004). These include changes in the inclination (and therefore, changes in the projected size $\dot{x} \equiv$ $\left.a_{p} / c d(\sin i) / d t\right)$ of the orbit, large variations in the orbital period $\left(\dot{P}_{B} \neq 0\right)$, and unmodeled and systematic trends in the residuals. As described above, the values for $\dot{x}$ and $\dot{P}_{B}$ are not significant (see Table 2), and the object times as well as an isolated MSP, with no unmodeled trends in the residuals despite the fact that our timing precision is much higher than for either PSR J0045-7319 or PSR B1259-63. In order to avoid eclipses and any measurable tidal effects, the companion has to be a compact object. The implications of this are discussed below.

\footnotetext{
${ }^{4}$ Because of the advance of periastron between the epoch of the parameters in Table 2 and this observation, superior conjunction occurred $15 \mathrm{~min}$ and $52.8 \mathrm{~s}$ after periastron, a difference of $1.3 \mathrm{~s}$ relative to the reference epoch.
} 


\subsection{What is the mass of the companion?}

For a system consisting of two compact objects, the observed $\dot{\omega}$ is due solely to the effects of general relativity. This allows an estimate of the total mass of the system: $2.453(14) \mathrm{M}_{\odot}$. Given the mass function, the pulsar mass cannot be larger than $1.50 \mathrm{M}_{\odot}$ and the companion mass must be larger than $0.96 \mathrm{M}_{\odot}$. For a median inclination of $60^{\circ}$, the mass of the pulsar is $1.350 \mathrm{M}_{\odot}$, a value that is fairly typical of the neutron stars with well-determined masses. In this case, the companion mass would be about $1.105 \mathrm{M}_{\odot}$.

For $49.76^{\circ}<i<52.86^{\circ}$, both components would have masses within the present range of well-measured neutron stars (see Fig. 1); however, given a flat probability distribution in $\cos i$, it is about 15 times more likely that $52.86^{\circ}<i<90^{\circ}$, where $m_{c}<1.2 \mathrm{M}_{\odot}$. In the higher inclination range, there is the possibility that the companion is the lightest NS ever discovered. However, this is probably more than compensated for in the lower inclination range by the possibility that the companion is a WD or a stripped core of a giant star with $m_{c}>1.2 \mathrm{M}_{\odot}$. We therefore believe that the probabilities are not far from 15 to 1 against the companion being a neutron star. Given the slight possibility, though, that the companion star could be a MSP, we searched several of the 350, 820, and $1950 \mathrm{MHz}$ observations for additional pulsations, but found none.

\section{Flux Density Variations}

In Table 2 we list the average flux densities at 350, 425, 820 and $1950 \mathrm{MHz}$, together with the number of observations averaged, we derive a spectral index of -2.65 . At $350 \mathrm{MHz}$, the flux density changes very significantly, from about 0.2 to $1.4 \mathrm{mJy}$. Even our brightest detection is significantly fainter than the average flux density reported in Paper I (3.4 $\pm 0.4 \mathrm{mJy}$ ). This could explain the discrepancy in reported spectral indices; in Paper I we use the nondetection at $610 \mathrm{MHz}$ to derive $\alpha_{S}<-3.4$. However, the GMRT flux density at $325 \mathrm{MHz}$ comes from imaging, so it is possible that there is extra unpulsed radio emission in the vicinity of the pulsar. However, we can not exclude the possibility of some lapse in our understanding of the sensitivity of these systems.

The timescale for the variations near periastron $\left(\tau_{p}\right)$ is of the order of $1000 \mathrm{~s}$; near apastron the flux density is seen to change very slowly and monotonically over several hours, only becoming significant from one day to the next (i.e. $\tau_{a} / \tau_{p}>10$ ).

The flux density variations are almost certainly not caused by diffractive scintillation. The scintillation bandwidth at $350 \mathrm{MHz}$ is (very roughly) $10-20 \mathrm{kHz}$ (Cordes and Lazio 2002), so our observations average over many hundreds of scintles in the $50 \mathrm{MHz}$ band- 
width. Furthermore, refractive scintillation will change the flux by no more than $~ 50 \%$, on timescales of $\sim 10^{7}$ seconds (Cordes and Lazio, 2002). Therefore, if the ISM between us and the cluster has everywhere a Kolmogorov spatial spectrum and is described, to within a few orders of magnitude, by the Cordes and Lazio model, then no large-amplitude variations in the flux density should be observed. Scintillation by a region or regions of particularly high ionized gas density could provide an explanation, we could alternatively be dealing with small eclipses caused by gas in the vicinity of the binary.

\subsection{Gas from the Companion Star}

Flux density variations of similar magnitude and duration have been observed for several eclipsing binary pulsars away from superior conjunction. The best documented examples are PSR J1740-5340, a MSP-main sequence binary located in the GC NGC 6397 (Ferraro et al. 2001), and PSR J1748-2446A, an eclipsing binary MSP located in the GC Terzan 5 (Lyne et al. 1990; Lyne et al. 2000) also known as Terzan 5 A. As in virtually all eclipsing binaries, the companions of these two pulsars are losing gas as a result of their interaction with the pulsar wind. Part of the observed flux density variations away from superior conjunction are due to secondary eclipses thought to be caused by gas clumps that remain in the vicinity of these binary systems; even though the precise mechanism of the modulation is not known. Many, but not all, of these secondary eclipses are "total" (i.e. the pulsars become completely undetectable).

The companion of NGC 1851A is much more massive than the companions of either PSR J1740-5340 or Terzan 5 A. No outgassing has ever been clearly detected by pulsar timing in systems where the companion is a massive white dwarf, and it has not been detected in NGC 1851A either (\$4). However, there could still be gas clumps in the vicinity of the system if the companion of NGC 1851A is outgassing at a lower level than what we can detect. This is a possibility if it is a stripped core of a giant star.

Unlike PSR J1740-5340 or Terzan 5 A, in NGC 1851A we detect no "total" eclipses caused by gas clumps at superior conjunction or away from it. Furthermore, a common feature of such eclipses is that they invariably affect the lower frequencies more strongly (e.g. longer duration eclipses), although in varying degrees for different binary systems. That is not observed in NGC 1851A, where the attenuation may be frequency dependent, although in a variable and inconsistent manner. However, it is important to note that the behavior of the other pulsars mentioned above varies strongly from orbit to orbit. In our case, we only have good observations through a single periastron and superior conjunction. With the current data we cannot conclusively demonstrate that the variations are caused by 
orbiting gas. However, we do consider it a possible explanation for the observed flux density variations.

\subsection{Refractive scintillation}

It is possible that, despite the predictions of Cordes and Lazio, the changes in flux density are indeed due to scintillation. This is more likely if, somewhere between us and the pulsar, there are unusual regions of the ISM acting as strong lenses. We can use the changes in $\tau$ to constrain their location. Assuming a maximum inclination of $90^{\circ}$, the pulsar is traveling at $v_{p} \sim 173 \mathrm{~km} \mathrm{~s}^{-1}$ relative to the center of mass at periastron, and at $v_{a} \sim 10 \mathrm{~km} \mathrm{~s}^{-1}$ relative to the center of mass at apastron. Furthermore, the distance and proper motion listed for this cluster in Table 1 imply a perpendicular cluster velocity of $\sim 160 \mathrm{~km} \mathrm{~s}^{-1}$.

If the lenses are near us, at apastron the velocity of the pulsar $v_{a}^{\prime}$ relative to those lenses can not be much different from the perpendicular velocity of the cluster times $f$ (the distance from the Solar System to the lens divided by the distance from the Solar System to NGC 1851). At periastron, we obtain $\left(20 \times f<v_{p}^{\prime}<320 \times f\right) \mathrm{km} \mathrm{s}^{-1}$, depending on how the pulsar's orbital velocity at periastron adds to its proper motion. This would imply $0.1<v_{p}^{\prime} / v_{a}^{\prime}=\tau_{a} / \tau_{p}<2$, which is inconsistent with observation. If $f$ is very small, then the Earth's orbital velocity becomes dominant in determining $\tau$ and we can then produce a larger range of $\tau_{a} / \tau_{p}$, however, no seasonal variations in $\tau_{a}$ are observable. If, on the other hand, the medium responsible for the variations has a small velocity relative to the center of mass of the binary, we obtain $\tau_{a} / \tau_{p} \sim f v_{p} / f v_{a}=(1+e) /(1-e)=16.85$, which is consistent with observation.

This suggests that a hypothetical lensing medium would probably be located in the cluster, which is also the case if we were instead observing gas clumps orbiting the binary itself. Given the proximity to the pulsar, we can then readily estimate the associated spatial scale of the medium, independently of its nature, from $v_{p}$ and $\tau_{p}$ : about $10^{5} \mathrm{~km}$.

\section{Conclusion and prospects}

In this work we have determined the phase-coherent timing solution of NGC 1851A. This allowed us to locate the pulsar in the globular cluster and determine precise rotational and orbital parameters. The geometry of the system, the lack of any observable tidal effects in the timing and the lack of eclipses or a measurable increase of the DM at superior conjunction allow us to exclude the possibility of an extended companion. This in turn allows us to 
calculate the total mass of the system from the observed rate of the advance of periastron: 2.453(14) $\mathrm{M}_{\odot}$. The pulsar mass has to be smaller than $1.5 \mathrm{M}_{\odot}$ and the companion mass has to be larger than $0.96 \mathrm{M}_{\odot}$. The companion is either a massive WD or the stripped core of a giant star, and less likely a neutron star.

The flux density variations remain puzzling. They could be due to gas clumps in the vicinity of the binary created by outgassing from the companion, a possibility considering that it could be the stripped core of a giant star. Alternatively, they could be due to refractive scintillation, in which case the lensing structures are also likely to be located in NGC 1851. This would imply the presence of intra-cluster gas, the second instance after 47 Tucanae (Freire et al. 2001) where such gas was confirmed in a globular cluster.

If we observe the pulsar often close to periastron, it might be possible to measure the velocity of the binary relative to the medium that causes the flux density variations and decide which of the scenarios discussed is the correct one. Furthermore, a detailed study of the orbital variability of $\tau$ might allow a determination of the orbital inclination of the system, and lead to a determination of the individual masses. So far, only one MSP, PSR J1909-3744 has a well-determined mass, 1.44 $\mathrm{M}_{\odot}$ (Jacoby et al. 2005). Measuring more MSP masses precisely is important in order to estimate how much mass a neutron star needs to accrete in order to become a MSP.

Such a detailed mass measurement will be confirmed (or refuted) by the measurement of another relativistic timing effect, the Einstein delay $(\gamma)$. Our simulations suggest that, with the present observing strategy and timing precision, we will be able to measure $\gamma$ to better than $1 \mathrm{~ms}$ within 6 to 8 years. For an edge-on orbit, $\gamma$ is predicted to be $16.13 \mathrm{~ms}$, and for $i=60^{\circ}, \gamma=19.44 \mathrm{~ms}$. The Shapiro delay can not be measured with the present timing precision, no tests of general relativity are possible with the present telescope sensitivities.

The National Radio Astronomy Observatory is a facility of the National Science Foundation operated under cooperative agreement by Associated Universities, Incorporated. We thank Jason Hessels and Fernando Camilo for help with observations, David Nice for assistance with TOA simulation software and Christopher Salter for a first skeptic review of the paper.

\section{REFERENCES}

Allen, C. W. and Cox, A. N.. (2000), "Allen's Astrophysical Quantities", AIP Press; Springer, New York 
Cordes, J. M. and Lazio, T. J. W. (2002), http://arxiv.org/abs/astro-ph/0207156

D’Amico, N., Possenti, A., Fici, L., Manchester, R. N., Lyne, A. G., Camilo, F., \& Sarkissian, J. 2002, ApJ, 570, L89

Damour, T. \& Deruelle, N. 1985, Ann. Inst. H. Poincaré (Physique Théorique), 43, 107

Damour, T. \& Deruelle, N. 1986, Ann. Inst. H. Poincaré (Physique Théorique), 44, 263

Dinescu, D. I., Girard, T. M., \& van Altena, W. F. 1999, AJ, 117, 1792

Damour, T. \& Taylor, J. H. 1992, 45, 1840

Dubath, P., Meylan, G., \& Mayor, M. 1997, A\&A, 324, 505

Faulkner, A. J. et al. 2005, ApJ, 618, L119

Ferdman, R. et al. 2006, Presented at the 208th AAS meeting.

Ferraro, F. R., Possenti, A., D’Amico, N., \& Sabbi, E. 2001, ApJ, 561, L93

Freire, P. C., Kramer, M., Lyne, A. G., Camilo, F., Manchester, R. N., \& D’Amico, N. 2001, ApJ, 557, L105

Freire, P. C., Camilo, F., Kramer, M., Lorimer, D. R., Lyne, A. G., Manchester, R. N., \& D'Amico, N. 2003, MNRAS, 340, 1359

Freire, P. C., Gupta, Y., Ransom, S. M., \& Ishwara-Chandra, C. H. 2004, ApJ, 606, L53

Freire, P. C. C., Hessels, J. W. T., Nice, D. J., Ransom, S. M., Lorimer, D. R., \& Stairs, I. H. 2005, ApJ, 621, 959

Freire, P. C. C. 2005, ASP Conf. Ser. 328: Binary Radio Pulsars, 328, 405

Harris, W. E. 1996, AJ, 112, $1487 . \quad$ Updated version at http://www.physics.mcmaster.ca/resources/globular.html

Jacoby, B. A., Hotan, A., Bailes, M., Ord, S., \& Kuklarni, S. R. 2005, ApJ, 629, L113

Kaplan, D. L., et al. 2005, PASP, 117, 643

Kuijken, K. \& Gilmore, G. 1989, MNRAS, 239, 571

Lai, D. 1996, ApJ, 466, L35 
Lyne, A. G., Johnston, S., Manchester, R. N., Staveley-Smith, L., \& D'Amico, N. 1990, Nature, 347, 650

Lyne, A. G., Mankelow, S. H., Bell, J. F., \& Manchester, R. N. 2000, MNRAS, 316, 491

Lorimer, D. R., 2005, Living Reviews in Relativity, vol. 8, no. 7

Lombardi, J. C., Jr., Proulx, Z. F., Dooley, K. L., Theriault, E. M., Ivanova, N., \& Rasio, F. A. 2006, ApJ, 640, 441

Phinney, E. S. 1992, Phil. Trans. Roy. Soc. A, 341, 39

Ransom, S. M., Hessels, J. W. T., Stairs, I. H., Freire, P. C. C., Camilo, F., Kaspi, V. M., \& Kaplan, D. L. 2005, Science, 307, 892

Ransom, S. M., Stairs, I. H., Backer, D. C., Greenhill, L. J., Bassa, C. G., Hessels, J. W. T., \& Kaspi, V. M. 2004, ApJ, 604, 328

Rasio, F. A., \& Shapiro, S. L. 1991, ApJ, 377, 559

Standish, E. M. 1998, JPL Planetary and Lunar Ephemerides, DE405/LE405, Memo IOM 312.F-98-048, (Pasadena: JPL). http://ssd.jpl.nasa.gov/iau-comm4/de405iom/de405iom.pdf

Salaris, M., \& Weiss, A. 1997, A\&A, 327, 107

Taylor, J. H. 1992, Philosophical Transactions of the Royal Society of London, A, 341, 117

Wang, N., Johnston, S., \& Manchester, R. N. 2004, MNRAS, 351, 599 
Table 1. Parameters for the globular cluster NGC 1851

\begin{tabular}{lcl}
\hline \hline Right Ascension of center, $\alpha$ & $05^{\mathrm{h}} 14^{\mathrm{m}} 06^{\mathrm{s}} 3$ & $($ Harris 1996) \\
Declination of center, $\delta$ & $-40^{\circ} 02^{\prime} 50$ & $($ Harris 1996) \\
Galactic longitude, $l\left(^{\circ}\right)$ & 244.51 & \\
Galactic latitude, $b\left(^{\circ}\right)$ & -35.04 & \\
Cluster distance, $D(\mathrm{kpc})$ & 12.1 & $($ Harris 1996) \\
Core radius, $\theta_{c}(\operatorname{arcmin})$ & 0.06 & $($ Harris 1996) \\
$v_{z}(0)\left(\mathrm{km} \mathrm{s}^{-1}\right)$ & 11.3 & (Dubath, Meyland \& Mayor 1997) \\
Proper Motion in $\alpha, \mu_{\alpha}\left(\operatorname{mas~yr}^{-1}\right)$ & $+1.28 \pm 0.68$ & (Dinescu et al. 1999) \\
Proper Motion in $\delta, \mu_{\delta}\left(\operatorname{mas~yr}^{-1}\right)$ & $+2.39 \pm 0.65$ & (Dinescu et al. 1999) \\
Age $(\mathrm{Gyr})$ & $8.9 \pm 0.9$ & (Salaris \& Weiss 1997) \\
\hline
\end{tabular}


Table 2. Parameters for the PSR J0514-4002A binary system

\begin{tabular}{|c|c|}
\hline \multicolumn{2}{|c|}{ Observation parameters and flux density } \\
\hline First observation (MJD) & 53258 \\
\hline Last observation (MJD) & 54066 \\
\hline Number of TOAs & 316 \\
\hline Residual rms $(\mu \mathrm{s})$ & 30 \\
\hline Epoch (MJD) & 53623.155088 \\
\hline Flux density, & \\
\hline$\ldots$ at $350 \mathrm{MHz}, S_{350}(\mathrm{mJy})$ & $0.62(2)(93)^{\mathrm{a}}$ \\
\hline$\ldots$ at $425 \mathrm{MHz}, S_{425}(\mathrm{mJy})$ & $0.28(6)(2)$ \\
\hline$\ldots$ at $820 \mathrm{MHz}, S_{820}(\mathrm{mJy})$ & $0.065(6)(12)$ \\
\hline$\ldots$ at $1950 \mathrm{MHz}, S_{1950}(\mathrm{mJy})$ & $0.0056(10)(3)$ \\
\hline Spectral index, $\alpha_{S}$ & $-2.71(9)$ \\
\hline \multicolumn{2}{|l|}{ Timing parameters } \\
\hline Right Ascension, $\alpha(\mathrm{J} 2000)$ & $05^{\mathrm{h}} 14^{\mathrm{m}} 06^{\mathrm{s}} \cdot 6927(2)$ \\
\hline Declination, $\delta(\mathrm{J} 2000)$ & $-40^{\circ} 02^{\prime} 48^{\prime \prime} 897(2)$ \\
\hline Spin frequency, $\nu(\mathrm{Hz})$ & $200.37770740529(11)$ \\
\hline Time derivative of $\nu, \dot{\nu}\left(10^{-17} \mathrm{~Hz}^{2}\right)$ & $-4.7(5)$ \\
\hline Dispersion Measure, DM $\left(\mathrm{pc} \mathrm{cm}^{-3}\right)$ & $52.1489(6)$ \\
\hline Orbital period, $P_{b}$ (days) & $18.78517915(4)$ \\
\hline Projected size or orbit, $x(\mathrm{l}-\mathrm{s})$ & $36.296588(9)$ \\
\hline Time of passage through periastron, $T_{0}$ (MJD) & $53623.1550879(4)$ \\
\hline Eccentricity, $e$ & $0.8879773(3)$ \\
\hline Longitude of periastron, $\omega\left(^{\circ}\right)$ & $82.266550(18)$ \\
\hline Rate of advance of periastron, $\dot{\omega}\left({ }^{\circ} \mathrm{yr}^{-1}\right)$ & $0.01289(4)$ \\
\hline Einstein delay, $\gamma(\mathrm{ms})$ & {$[8 \pm 26]^{\mathrm{b}}$} \\
\hline Time derivative of $x, \dot{x}\left(10^{-12} \mathrm{l}-\mathrm{s} / \mathrm{s}\right)$ & {$[-0.1 \pm 0.4]$} \\
\hline Time derivative of $P_{B}, \dot{P_{B}}\left(10^{-9}\right)$ & {$[1.5 \pm 0.7]$} \\
\hline \multicolumn{2}{|l|}{ Derived parameters } \\
\hline Angular distance from center of cluster, $\theta_{\perp}$ & \\
\hline$\ldots$ (arcseconds) & 4.6 \\
\hline$\ldots$ (core radii $)$ & 1.3 \\
\hline$\ldots(\mathrm{pc})$ & 0.27 \\
\hline Spin period, $P(\mathrm{~ms})$ & $4.990575114114(3)$ \\
\hline Time derivative of $P, \dot{P}\left(10^{-21}\right)$ & $1.17(14)$ \\
\hline Maximum cluster acceleration, $a_{c, \max }\left(\mathrm{m} . \mathrm{s}^{-2}\right)$ & $18.4 \times 10^{-9}$ \\
\hline Maximum intrinsic $\dot{P}, \dot{P}_{\text {int }, \max }\left(10^{-19}\right)$ & 3.0 \\
\hline Maximum magnetic flux density, $B_{0}\left(10^{9}\right.$ Gauss $)$ & 1.2 \\
\hline Minimum characteristic age, $\tau_{c, \min }(\mathrm{Gyr})$ & 0.26 \\
\hline Mass function, $f\left(\mathrm{M}_{\odot}\right)$ & $0.14549547(11)$ \\
\hline Total system mass, $\mathrm{M}\left(\mathrm{M}_{\odot}\right)$ & $2.453(14)$ \\
\hline
\end{tabular}




\section{Table 2-Continued}

Maximum pulsar mass, $\mathrm{M}_{p, \max }\left(\mathrm{M}_{\odot}\right) \quad 1.50$

Minimum companion mass, $\mathrm{M}_{c, \min }\left(\mathrm{M}_{\odot}\right) \quad 0.96$

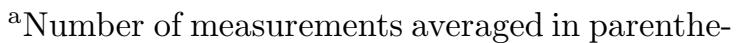
ses. Flux uncertainty is $30 \% / \sqrt{N}$

${ }^{b}$ Values in square brackets are not 3- $\sigma$ significant, nor are they expected to be so. They were not fit when determining the remaining timing parameters. 
Table 3. Geometric parameters for the companion of NGC 1851A

\begin{tabular}{lllll}
\hline$i$ & $m_{c}$ & $a_{\text {sup }}$ & $S=a_{\text {sup }} \cos i$ & $R_{c}$ \\
$\left({ }^{\circ}\right)$ & $\left(\mathrm{M}_{\odot}\right)$ & $\left(\mathrm{R}_{\odot}\right)$ & $\left(\mathrm{R}_{\odot}\right)$ & 0.82 \\
\hline 90 & 0.8483 & 4.2471 & 0 & 0.83 \\
85 & 0.8528 & 4.2502 & 0.3704 & 0.84 \\
80 & 0.8665 & 4.2596 & 0.7397 & 0.86 \\
75 & 0.8901 & 4.2758 & 1.1067 & 0.89 \\
70 & 0.9252 & 4.2996 & 1.4705 & 0.93 \\
65 & 0.9739 & 4.3322 & 1.8309 & 0.99 \\
60 & 1.0396 & 4.3754 & 2.1877 & \\
\hline
\end{tabular}




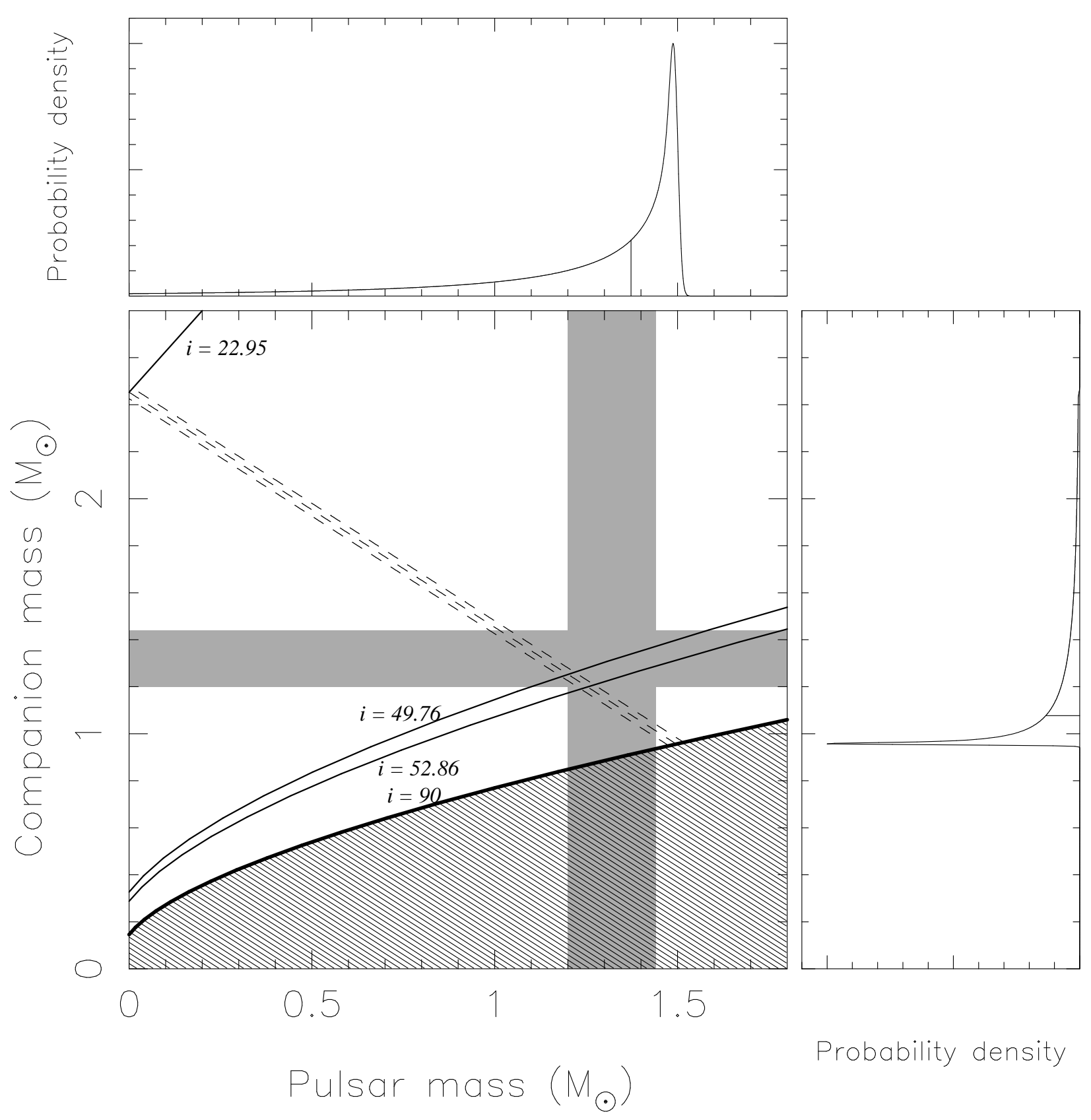

Fig. 1.- Constraints on the masses of NGC 1851A and its companion. The hatched region is excluded by knowledge of the mass function and by $\sin i \leq 1$. The diagonal dashed lines correspond to a total system mass that causes a general-relativistic $\dot{\omega}$ equal or within $2-\sigma$ of the measured value. The four solid curves indicate constant inclinations. We also display the probability density function for the mass of the pulsar (top) and the mass of the companion $(r i g h t)$, and mark the respective medians with vertical (horizontal) lines. 